\title{
ESTADO DE DERECHO Y CONTROL JURISDICCIONAL: DESAFÍOS Y TENSIONES ${ }^{*}$
}

\author{
Gonzalo CANDia FalCón** \\ FRANCISCO JAVIER URBINA ${ }^{* * *}$
}

RESUMEN: El presente trabajo explica como la revisión judicial de la conformidad a derecho de los actos de una autoridad puede ser disruptiva para la vigencia y aplicación del mismo derecho, así como también para su capacidad de limitar la discrecionalidad de la autoridad. Ello por cuanto dicha actuación judicial puede impactar negativamente el sistema jurídico desde la perspectiva de las exigencias del Estado de Derecho, las cuales representan un estándar de actuación para todo tribunal de justicia. El artículo distingue dos exigencias fundamentales constitutivas de la idea de Estado de Derecho, las cuales están en juego en el control jurisdiccional de los actos de la autoridad, y demuestra como dicho control puede satisfacer una de esas exigencias, mientras al mismo tiempo impacta negativamente el cumplimiento de la otra. Se ilustra lo anterior mediante el análisis de un caso reciente: el recurso de protección interpuesto en contra de la decisión de la CEA de Atacama, que aprobó el Estudio de Impacto Ambiental del proyecto minero El Morro.

PALABRAS CLAVE: Estado de Derecho - revisión judicial - adjudicación.

\section{Rule OF LAW AND JUdicial REVIEW: CHALlENGES AND TENSIONS}

ABSTRACT: This article seeks to explain how judicial review of administrative action can have a negative impact in the application of the law itself as well as in its capacity to limit the discretion of public author-

\footnotetext{
* $\quad$ Fecha de recepción: 9 de septiembre de 2015.

Fecha de aceptación: 19 de mayo de 2016.

** Doctor en Derecho (S.J.D), Universidad de Georgetown. Profesor de Derecho Constitucional y Derecho Internacional de los Derechos Humanos, Facultad de Derecho, Pontificia Universidad Católica de Chile. Correo electrónico: gfcandia@uc.cl

*** Doctor en Derecho (D.Phil.), Universidad de Oxford. Profesor de Derecho Constitucional e Introducción al Derecho, Facultad de Derecho, Pontificia Universidad Católica de Chile. Correo electrónico: furbina@uc.cl. Los autores agradecen los excepcionalmente útiles, precisos y detallados comentarios proveídos por los dos árbitros del presente artículo. Las traducciones al español de textos en inglés en este artículo son de los autores.
} 
ities. This transpires when courts adjudicate cases ignoring the demands of the rule of law, which represent a standard of action for any court. Two basic demands of the rule of law are distinguished here and both are at stake when courts review decisions made by public authorities. The article also explains how the jurisdictional control can satisfy one of those demands and at the same time compromising the capacity of the law for satisfying the other. The authors illustrate their point by analyzing a relatively recent judgment from the Chilean Supreme Court concerning a "writ of protection" that challenged the decision of the CEA of Atacama which approved the Study of Environmental Impact of the mining project El Morro.

KEYWORDS: Rule of law - judicial review - adjudication.

Sumario: Introducción; 1) Estado de Derecho y control jurisdiccional; 2) Dos concepciones del Estado de Derecho: Fuller y Dicey; 3) Estado de Derecho y control jurisdiccional de la autoridad: tensiones; 4) Mejorar o empeorar la institucionalidad como estándar de las decisiones judiciales; 5) Ilustración: Caso el Morro II. Conclusión. Bibliografía citada.

\section{INTRODUCCIÓN}

El control jurisdiccional de los actos de las autoridades públicas parece a primera vista como necesariamente consistente y necesario para la vigencia del Estado de Derecho. Desde la perspectiva de la institucionalidad jurídica, parece evidente que la intervención judicial que precisamente verifica la sujeción de una autoridad a dicha institucionalidad es necesariamente favorable al respeto y vigencia de esta. Reconociendo la importancia que puede tener la actuación jurisdiccional para sujetar a la autoridad al derecho, este artículo, sin embargo, plantea que esa misma actuación jurisdiccional puede llegar a ser también perjudicial para la institucionalidad que regula a una determinada autoridad.

En ese sentido, el objetivo principal de este trabajo es demostrar como el control jurisdiccional de los actos de las autoridades puede ser disruptivo para el mismo derecho cuyo cumplimiento se controla y, por lo tanto, como ese tipo de decisiones puede impactar negativamente en el potencial que tiene el derecho para limitar la discrecionalidad de la autoridad. Dada que la existencia de normas jurídicas que ordenan la conducta de una autoridad, y la consiguiente limitación de la discrecionalidad de la misma, son comúnmente entendidas como exigencias paradigmáticas 
de la idea de Estado de Derecho, es posible presentar nuestras conclusiones en esos términos. Así, se puede decir que el objetivo de este trabajo es demostrar como el control jurisdiccional de los actos de las autoridades puede ser perjudicial para el Estado de Derecho.

Para cumplir ese objetivo, el artículo expondrá dos nociones constitutivas del ideal de Estado de Derecho. Ambas nociones plantean ciertas exigencias respecto del orden jurídico y la conducta de las autoridades. Al hacer explícitas estas exigencias y su sentido, se podrá comprender de manera precisa la posible tensión entre la acción de los tribunales, por un lado, y la vigencia del derecho y su capacidad de limitar la discrecionalidad de las autoridades, por otro. En ese contexto, se especificará, en términos generales, cuándo una decisión judicial es consistente con las ideas constitutivas de la idea de Estado de Derecho y cuándo no lo es. En este respecto, el artículo propone un estándar para las actuaciones de los tribunales de justicia. El artículo ilustrará el argumento teórico expuesto a partir del análisis de una sentencia dictada por la Corte Suprema chilena en 2014. Ella recae sobre un recurso de protección interpuesto contra la Resolución de Calificación Ambiental de la Comisión de Evaluación Ambiental de Atacama que aprobó el Estudio de Impacto Ambiental del proyecto minero El Morro, el cual fue visto en apelación por la Corte Suprema (2014). El argumento queda mejor explicado tras el análisis de los detalles de este caso concreto. El caso en cuestión fue elegido por los autores en la medida que el mismo es revelador del problema que se presenta en este artículo - permitiendo mostrar como dicho problema se puede manifestar en decisiones concretas de nuestros tribunales-. En la sentencia en comento, se argumentará, existe una deficiencia en la forma (no necesariamente en el fondo) de la decisión judicial que impacta negativamente en la institucionalidad desde la perspectiva de las exigencias planteadas por el ideal del Estado de Derecho, con las consecuencias indeseables que de ello se derivan.

\section{1) ESTADO DE DERECHO Y CONTROL JURISDICCIONAL}

El control jurisdiccional de los actos de la autoridad tiende a ser interpretado como inequívocamente favorable o consistente con las exigencias propias del Estado de Derecho. Esta idea, como veremos, es de larga data, y hay un vínculo estrecho entre control jurisdiccional de la acción de la autoridad, típicamente de la autoridad administrativa, y la 
limitación de la misma por el derecho ${ }^{1}$. Sin embargo, así como existe este vínculo, existe también una tensión que no ha sido debidamente ponderada por la doctrina. En efecto, la acción de los tribunales también podría ser contraria a las exigencias del Estado de Derecho bajo ciertas circunstancias específicas. Tal como se indicó, este aspecto del control jurisdiccional tiende a ser pasado por alto, y por lo mismo, exige una mayor elaboración.

La idea de Estado de Derecho es compleja ${ }^{2}$. De hecho, existen diversas visiones acerca de las demandas propias del Estado de Derecho ${ }^{3}$. Así, por ejemplo, autores como Jeremy Waldron distinguen al menos tres aproximaciones al concepto: (a) formal, (b) sustantiva y (c) procedimen$\mathrm{tal}^{4}$. Sin embargo, pese a la diversidad de posiciones sobre el tema, todas ellas coinciden en que la idea de Estado de Derecho implica, al menos, la sujeción de la autoridad, cualquiera sea esta, a las exigencias del derecho. Asimismo, como elaboraremos posteriormente, para que esa sujeción sea efectiva, el derecho aplicable debe poseer ciertas características que permitan que quienes están sujetos a él puedan ordenar su acción de forma tal de cumplir con el mismo. Esta idea también es ampliamente compartida $y$, si bien no siempre es articulada, al menos es compatible con las dis-

Para una descripción histórica de la evolución del proceso que terminó por sujetar a la autoridad administrativa al poder del derecho en Chile, véase: Bordalí, Andrés (2006) "La administración pública ante los tribunales de justicia chilenos". Revista Chilena de Derecho, Vol. 33, N 1, pp. 17-36. Desde la aproximación propia del principio de juridicidad, véase: Soto-Kloss, Eduardo (1996) Derecho Administrativo - Bases Fundamentales. Santiago de Chile: Editorial Jurídica de Chile, Tomo II, 486 pp., pp. 24-43. En el mismo sentido, véase: CEA, José Luis (2005) "Estado constitucional de derecho: nuevo paradigma jurídico". Anuario Latinoamericano de Derecho Constitucional, Año 11, pp. 43-56. Si bien la doctrina chilena no refiere mayoritariamente el estudio de la noción de Estado de Derecho, ella sí lo hace de forma indirecta al momento de tratar cuestiones específicas, e.g: NúNEEZ, Juan Ignacio (2011) "Vigencia del dogma de la irresponsabilidad del estado legislador en el estado constitucional de derecho. Ensayando bases para una teoría sobre la responsabilidad patrimonial del estado legislador". Estudios Constitucionales, Año 9, N 1, pp. 217-230 o Nogueira, Humberto (2009) "Los derechos económicos, sociales y culturales como derechos fundamentales efectivos en el constitucionalismo democrático latinoamericano". Estudios Constitucionales, Año 2, Nº 7, pp. 143-205.

2 Tamanaha, Brian (2004) On the rule of law. History, politics, theory. Cambridge: Cambridge University Press, 180 pp., p. 91. En el contexto de la discusión sobre el contenido del Estado de Derecho, se hace necesario también relacionar la idea anglosajona de Rule of Law con la noción alemana de Rechstaat. En relación a esto último, véanse los trabajos que forman parte de: Silkenat, James R.; Hickey, James E.; y Barenboim, Peter D. (editores) (2014) The legal doctrines of the rule of law and the legal state (Rechtsstaat). Heidelberg: Springer, $367 \mathrm{pp}$.

3 Craig, Paul (1997) "Formal and substantive conceptions of the rule of law: an analytical framework”. Public Law, N467, pp. 467-487.

4 WALDRON, Jeremy (2011) "The rule of law and the importance of procedure". En Fleming, James E. (editor): Getting to the Rule of Law. Nueva York: New York University Press, 298 pp., pp. 4-5. 
tintas aproximaciones al concepto de Estado de Derecho. Estas dos ideas -la sujeción de la autoridad a derecho y la necesidad de la producción de un derecho sujeto a ciertos estándares de calidad técnica- pueden ser caracterizadas como constitutivas de una concepción formal de Estado de Derecho ${ }^{5}$. Mas se debe hacer notar que la concepción formal de Estado de Derecho no se opone a las otras visiones, pues estas suponen la existencia de un piso común -la concepción formal-al que agregan más elementos, como, por ejemplo, el respeto por los derechos fundamentales sustantivos o el gobierno democrático. También se debe notar que las dos ideas recién mencionadas -la sujeción de la autoridad a derecho y la necesidad de la producción de un derecho sujeto a ciertos estándares de calidad técnica- constituyen estándares razonables en sí mismos, y generalmente aceptados, independientes del concepto (sea Estado de Derecho u otro) bajo el cual se les pretenda subsumir. Así, aquí tratamos ambas ideas bajo el concepto de Estado de Derecho, pues comúnmente se las asocia a este. Sin embargo, no hay nada de especial relevancia en el término Estado de Derecho, y nada de nuestro argumento depende de que a estas ideas se las trate bajo ese nombre o bajo cualquier otro.

El presente trabajo buscará desagregar analíticamente las dos ideas anteriormente señaladas, y notar una posible tensión entre ambas. Dicha tensión, se argumentará, se produce cuando, en la realización institucional de un aspecto del Estado de Derecho se sacrifica otro de los aspectos, sacrificio que a su vez debilita al primero. Esto es lo que explicaremos en la sección a continuación.

\section{2) DOS CONCEPCIONES DEL ESTADO DE DERECHO: FULLER Y DICEY}

Tal como se mencionó anteriormente, hay al menos dos ideas fundamentales que típicamente son asociadas a la idea de Estado de Derecho ${ }^{6}$. La primera es la limitación de la discrecionalidad del poder, y en particular de la autoridad administrativa o de gobierno, mediante el derecho. La segunda se relaciona con la idea de un sistema jurídico "en forma”, capaz

5 En este sentido, incluso defensores de la idea de un Estado de Derecho sustantivo aceptan que el piso mínimo que configura todo Estado de Derecho son las mencionadas exigencias, propias de la legalidad formal. Véase: Bingham, Tom (2011) The Rule of Law. Londres: Penguin Books, 230 pp., pp. 17-18.

6 En esta subsección y la siguiente seguimos y desarrollamos, con variadas enmiendas y complementos, lo que uno de los autores expresó en: Urbina, Francisco Javier y Urbina, Ignacio (2015) "Revisión judicial de la sanción ambiental (A propósito de Pascua Lama)". En Fermandois, Arturo y García, José Francisco (editores): Sentencias Destacadas 2014. Santiago de Chile: Libertad y Desarrollo, 377 pp., pp. 61-89 y pp. 71-79. 
de ordenar eficazmente ciertas acciones humanas en base a reglas. Si bien estas ideas han sido expresadas por diversos autores, para fijar los términos, nos centraremos en la exposición que han hecho de ellas quienes le han dado a cada una su formulación más canónica e influyente: Albert Venn Dicey, a la primera, y Lon Fuller, a la segunda. Metodológicamente, la elección de estos dos autores se justifica en la necesidad de distinguir cada una de estas ideas de otras relacionadas para su tratamiento por separado, con el objetivo de poder observar más claramente su interacción y notar posibles tensiones que han pasado desapercibidas. Cada uno de los autores mencionados enfoca su trabajo sobre Estado de Derecho de manera especialmente acentuada en una de las ideas específicas. En efecto, Dicey centra su tratamiento del Estado de Derecho en la idea de la sujeción de la autoridad a normas jurídicas, mientras que Fuller elabora la idea de un derecho que satisfaga ciertas exigencias que le permitan ordenar la conducta humana. Por cierto, ambos autores notan las relaciones que hay entre la idea específica del Estado de Derecho en que enfocan su trabajo y otras ideas relacionadas. Mas ambos articulan con notable claridad un aspecto determinado y específico del Estado de Derecho, y su tratamiento de él es canónico en la literatura internacional. Así enfocarnos en estos autores es una manera de delimitar con precisión las ideas mencionadas y de observar sus relaciones. Esto es especialmente necesario respecto de un concepto que, como dijimos, es complejo y generalmente es concebido como incluyendo una variedad de exigencias las que no siempre son claramente distinguidas. Así, existe un riesgo siempre presente de confusión y falta de claridad analítica respecto de los diversos elementos de la idea de Estado de Derecho, con el infeliz resultado de hacer más improbable la reflexión sobre la interacción entre los distintos elementos, y en particular las tensiones entre algunos de ellos.

La primera de las ideas de Estado de Derecho mencionadas, la sujeción de la autoridad al derecho ${ }^{7}$, tiene su expresión paradigmática y tal vez más difundida en la obra de A.V. Dicey, el constitucionalista más célebre de la Inglaterra victoriana y eduardiana. Para Dicey, una de las características básicas de la constitución inglesa era, junto con la soberanía del parlamento y las "convenciones de la Constitución”, el "imperio del derecho" (rule of law) ${ }^{8}$. La idea central en la noción de imperio del derecho en Dicey es la limitación de la discrecionalidad de las autori-

7 En relación a este punto, véase también en la doctrina nacional: SiLva Bascuñán, Alejando (1997) Tratado de Derecho Constitucional. Principios, Estado y Gobierno. Tomo I. Santiago de Chile: Editorial Jurídica de Chile, 588 pp., pp. 240-243.

8 Dicey, A.V. (2013a) The law of the constitution. Oxford: Oxford University Press, 572 pp., pp. 95-184. Aunque Dicey pensaba que este aspecto no era privativo de la constitución inglesa, pues el constitucionalismo de los Estados Unidos también otorgaba un lugar central 
dades por el derecho. Para Dicey el caso paradigmático de lo anterior está representado por la imposición de sanciones. En efecto, ellas solo se pueden establecer cuando existe "una infracción precisa a la ley" . En esto se manifiesta lo que Timothy Endicott ha llamado "el mejor aspecto de una preocupación por el Estado de Derecho: la determinación de no someter a las partes y a la comunidad a la voluntad arbitraria de un funcionario"10. Las personas no están sujetas a la mera voluntad de otra persona que está en autoridad, sino fundamentalmente al derecho, el cual es aplicado por la autoridad, a la cual sirve de fundamento y límite. Por lo mismo, Dicey enfatiza que otro aspecto central del Estado de Derecho es que el derecho vincula igualmente a autoridades y a gobernados, y es administrado por tribunales ordinarios de justicia ${ }^{11}$.

Este último punto es fundamental en la noción de imperio del derecho de Dicey, y, a su entender, representa uno de los elementos que distinguían favorablemente el orden constitucional inglés de aquel propio de las "naciones continentales". En Inglaterra no solo están las autoridades sujetas al derecho, sino que además todos, autoridades y gobernados, se encuentran sujetos a un mismo derecho -lo que Dicey llama "igualdad legal"12 - Este derecho es aplicado por los tribunales ordinarios a autoridades y gobernados por igual y, crucialmente, dichos tribunales son independientes de la administración ${ }^{13}$. En este régimen descrito por Dicey, cualquier persona puede reclamar de los actos de la autoridad frente a tribunales y la autoridad deberá demostrar la conformidad de su actuar con el derecho vigente, sin contar con un estatuto privilegiado.

Un aspecto interesante de la noción de imperio del derecho en Dicey es que para él es de enorme importancia que los principios del imperio del derecho están encarnados en el derecho concreto inglés, elaborado a partir de "fallos judiciales que determinan los derechos de personas

al rule of law. Véase: Dicey, A.V. (2013b) Comparative constitutionalism. Oxford: Oxford University Press, 402 pp., p. 78.

9 Dicey (2013a) 97.

10 Endicotr, Timothy (2012) "Legal interpretation". En Marmor, Andrei (director): The Routledge companion to philosophy of law. New York: Routledge University Press, 656 pp., pp. 109-122 y p. 110.

11 DiceY (2013a) 100

12 DiceY (2013a) 100.

13 Dicey (2013a) 100-115. El contraste es, por cierto, con el derecho administrativo francés, el cual para Dicey establecía un estatuto separado para ciudadanos y administración, y, crucialmente, establecía tribunales administrativos que eran parte de la misma administración, no del poder judicial, para la reclamación de los actos de la administración. Dicey nota una evolución en las instituciones francesas de su época. Ver su detallada discusión del modelo francés en el capítulo XII (incorporado en la tercera edición de su The Law of the Constitution, y revisado en la séptima) de Dicey (2013a) 235-249 (para la versión de la tercera edición) y pp. 368-409 (para la versión revisada de la séptima). 
privadas en casos particulares conocidos por las corte" 14 . Este derecho, con que los jueces contrastarán la acción de la autoridad, no consiste en "las declaraciones o definiciones de derechos tan queridas por los constitucionalistas extranjeros" 15 , sino en una serie de categorías jurídicas relativamente concretas, que son generalizaciones de lo resuelto por los tribunales en casos anteriores ${ }^{16}$. Al ser un derecho elaborado a partir de casos, este no está expresado en términos de declaraciones de derechos relativamente abstractos, sino en términos de "remedies" -acciones judiciales que dan títulos precisos para reclamar algo concreto en un tribunal ${ }^{17}$-. Por cierto, como admite Dicey, el derecho elaborado a partir de casos judiciales no es el único que puede ser preciso y aplicable, y así garantizar los derechos de las personas ${ }^{18}$.

Si bien la doctrina de Dicey acerca del rule of law no lo trata explícitamente, ella supone ciertos estándares que debe cumplir el derecho, pues la sujeción de la autoridad al derecho resulta ilusoria si el derecho que sujeta a la autoridad no tiene ciertas características. Además de otorgar acciones ante tribunales y de establecer exigencias sustantivas a la autoridad, las exigencias del derecho deben tener cierta precisión y concreción para su recta operación en el sistema jurídico. La contrapartida de esto es que la sujeción de la autoridad (y de los ciudadanos) al derecho se hace más precaria cuando este es excesivamente abstracto, vago, u oscuro. De ser así, no podrá guiar a la autoridad, ni la sujetará a un estándar deseable común y conocido. Ni la autoridad ni el ciudadano sabrán qué es lo que exige el derecho, y se reemplazará una discrecionalidad o arbitrio (el de la autoridad) por otro (el del juez).

Esto lleva al análisis de la segunda idea de Estado de Derecho mencionada anteriormente. Su expositor más destacado es el jurista norteamericano Lon Fuller. Para Fuller el derecho es "la tarea de someter la conducta humana al gobierno de las reglas" 19 . Por cierto, no se trata de manejar o manipular las acciones de los ciudadanos. El mismo Fuller explica que hay una "diferencia básica entre la dirección gerencial y el derecho: el derecho no es un asunto, como el management, de dirigir a otras personas respecto de como realizar tareas designadas por un superior, sino básicamente concierne el proveer a la ciudadanía de una estructura sólida

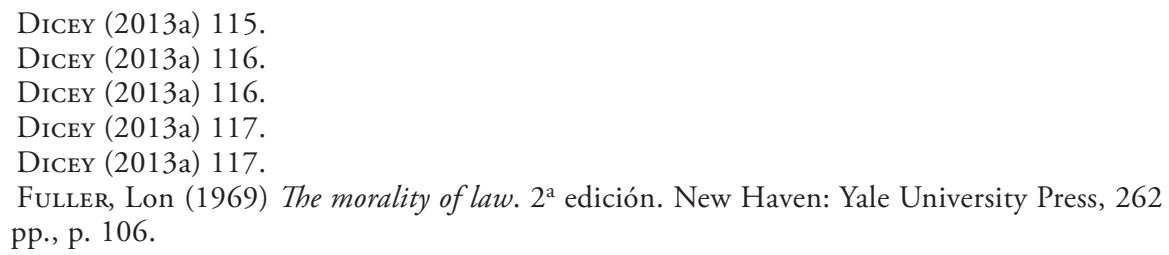


y estable para sus interacciones con otros; el rol del Estado [government] sería el ser el guardián de la integridad de ese sistema" ${ }^{20}$.

Para que el derecho sea exitoso en su tarea, es necesario que el ordenamiento jurídico reúna una serie de características fundamentales ${ }^{21}$. Estas son los llamados desiderata ("deseables" en latín) del Estado de Derecho. Ellos son: generalidad, claridad, publicidad, prospectividad, estabilidad, coherencia, posibilidad de ser obedecido, y congruencia entre el derecho y su aplicación por las autoridades pertinentes ${ }^{22}$. Que el ordenamiento jurídico cumpla estos desiderata es importante pues solo así podrá imponer exigencias razonables a sus destinatarios, y de esta forma guiar racionalmente el actuar de los mismos. Si el derecho no cumple con estos desiderata, entonces es un derecho que es deficiente en relación a su capacidad para orientar la acción a través de reglas. Por ejemplo, si las reglas no son relativamente claras, entonces sus destinatarios no pueden saber exactamente qué es lo que exige el derecho de ellos. Asimismo, si no son coherentes y existen abundantes reglas contradictorias y no existe una solución institucional a la contradicción, entonces los destinatarios no pueden saber cuál regla deben seguir. En ambos casos, no podrán orientar efectivamente su acción en base a las reglas en cuestión.

Por cierto, estas no son exigencias absolutas y el derecho puede operar bien con algún nivel de, por ejemplo, contradicción interna o incerteza respecto de la exigencia de una regla algo ambigua. Pero los grados son importantes, y es deseable alcanzar el ideal lo más posible. Un derecho que no cumpla en absoluto con estos desiderata simplemente no será un derecho de reglas: será un derecho estructurado en base a la discreción. Sostiene Fuller que " $[\mathrm{u}] \mathrm{n}$ fracaso total en cualquiera de estos ocho sentidos [los relativos a los ocho desiderata] resulta no solo en un mal sistema jurídico; resulta en algo que no puede ser llamado sistema jurídico en absoluto" 23 .

Aquí no nos concierne determinar cuál es la forma correcta de llamar o catalogar un ordenamiento jurídico que no cumpla con los desiderata del Estado de Derecho, sino fundamentalmente entender cuáles son sus deficiencias. Como se sabe, contar con un orden jurídico complejo cuyas prescripciones desarrollan en términos generalmente aceptables los desiderata es fundamental para la vida en sociedad. En efecto, ello permite esta-

\footnotetext{
20 FULlER (1969) 210.

21 Fuller (1969) 96. Fuller compara los desiderata con reglas de carpintería, es decir, con estándares para la realización competente o excelente de una tarea en particular. Fuller (1969) 33-91. FULLER (1969) 39.
} 
bilizar las expectativas de las personas y agrupaciones, lo que resulta crucial para que ellas puedan desarrollar proyectos y planes de vida de largo plazo $^{24}$. Joseph Raz ha visto en este aspecto del Estado del Derecho una exigencia de la dignidad humana, pues "[r] espetar la dignidad humana implica tratar a los seres humanos como personas capaces de planificar y ordenar su futuro" 25 . También hace posible que el derecho pueda cumplir eficazmente su rol de coordinar la acción humana y así, por ejemplo, establecer extensos y complejos esquemas de coordinación social (tales como, por ejemplo, una institucionalidad tributaria, una regulación del tránsito, o una institucionalidad ambiental), los cuales son de enorme importancia para la vida en sociedad y que solo el derecho puede generar ${ }^{26}$. Asimismo, un orden jurídico que cumple estos desiderata otorga más garantías a los ciudadanos, toda vez que les permite entender adecuadamente qué es lo que el derecho exige de ellos y de la autoridad, y por lo tanto describe límites claros al actuar legítimo de $\operatorname{todos}^{27}$. Por último, el cumplimiento de los desiderata hace explícitas las pautas del actuar de la autoridad, y por lo tanto facilita su crítica ${ }^{28}$. En definitiva, la determinación del derecho que otorgan los desiderata permiten precisamente realizar aquel aspecto que aparece como un elemento particularmente virtuoso del Estado de Derecho: el que la relación entre autoridades y gobernados no sea una de sometimiento de los segundos a la voluntad irrestricta de los primeros, ni a su subjetividad, sino a la coordinación de ambos para el bien común en base a normas comunes.

Este último punto conecta directamente las dos nociones de Estado de Derecho. La autoridad se sujeta al derecho, pero la mejor forma de sujetar a la autoridad al derecho es mediante un derecho cuyas exigencias sean suficientemente precisas, claras, estables, prospectivas, etc. Es decir, un derecho que cumpla los desiderata. Un derecho de esas características tiene dos ventajas cruciales. En primer lugar, permite a las autoridades y a los gobernados sujetar su acción al derecho (hacen posible que la acción

\footnotetext{
24 Finnis, John (2011a) Natural law and natural rights. 2a edición. Oxford: Oxford University Press, 512 pp., pp. 272-273.

25 Raz, Joseph (1979) "The rule of law and its virtue". En Raz, Joseph (autor): The authority of law: essays on law and morality. Oxford: Oxford University Press, 292 pp., pp. 210-229.

26 El rol del derecho en solucionar problemas de coordinación social es central para Finnis. Véase, por ejemplo, su definición de derecho en: Finnis (2011a) 276. Para un tratamiento más extenso del problema de la coordinación, véase: Finnis, John (2011b) "Law as coordination”. En Finnis, John (autor): Collected essays of John Finnis. Volume IV, Oxford: Oxford University Press, 509 pp., pp. 66-73. También: Raz (1979) 342-346.

27 Sobre esto, véase los comentarios de Fuller sobre "reciprocidad" entre particulares y autoridad en: Fuller (1969) 39-40.

28 Fuller (1969) 157-59.
} 
humana esté guiada por las reglas del derecho). En segundo lugar, en términos de poder, presenta límites claros y conocidos a la discreción de la autoridad y, por lo mismo, límites que serán más fácilmente conocidos, así como también custodiados, al ser más clara su infracción. Ambas ventajas, como se ha dicho, poseen un enorme potencial tanto en términos de efectividad del derecho como de generar condiciones sociales que facilitan el bien de las personas y la justicia en las relaciones entre autoridades y gobernados.

\section{3) ESTADO DE DERECHO Y CONTROL JURISDICCIONAL DE LA AUTORIDAD: TENSIONES}

Con todo, el papel que representan los tribunales en este punto es más problemático de lo que sugiere la caracterización de Dicey. El papel clásico que se atribuye a los tribunales al hablar de Estado de Derecho es el de revisar los actos de la autoridad: que se pueda reclamar a un tribunal imparcial e independiente cuando una autoridad se aparta del derecho ${ }^{29}$. Esto es lo que exige la primera idea asociada a la noción de Estado de Derecho y es central en la teoría de Dicey. Pero si tenemos en mente el tipo de consideraciones que incorpora la segunda idea, entonces el rol de los tribunales se hace más problemático. El objetivo aquí es preservar un orden jurídico "en buena forma" 30 , es decir, un orden que satisfaga los desiderata. Lo que interesa destacar es que la intervención judicial puede ser beneficiosa o perjudicial para alcanzar este objetivo asociado a la segunda idea de Estado de Derecho. Al hacerlo, también promueve o perjudica la primera idea asociada al Estado de Derecho.

Una sentencia judicial realiza dos cosas conceptualmente distinguibles, aunque comúnmente implicadas una en la otra en la realidad. Por un lado aplica el derecho, y por otro, desarrolla el derecho. En el caso ideal, ambas realizan las exigencias del Estado de Derecho mencionadas arriba. En efecto, al aplicar el derecho, el tribunal sujeta efectivamente a la autoridad al ordenamiento jurídico; el tribunal reconoce y reafirma tanto el derecho como el hecho de que este vincule a la autoridad; este no es letra muerta, sino un límite real a la autoridad.

29 Dentro de la doctrina nacional, la conexión entre Estado de Derecho y control jurisdiccional ha sido tratada particularmente por Pablo Rodríguez. Véase: Rodríguez, Pablo (1990) Teoría de la interpretación jurídica. Santiago de Chile: Editorial Jurídica de Chile, 182 pp., pp. 11-14.

30 Finnis (2011a) 270. Es utilizada también por: Webber, Gregoire (2013) "Rights and the rule of law in the balance”. Law Quarterly Review, Vol. 129, pp. 399-419, p. 399. 
Mediante la sentencia el tribunal también típicamente desarrolla el derecho. Esto no ocurre solamente en los sistemas jurídicos del common law, en los que las decisiones de los tribunales vinculan a otros tribunales respecto de casos posteriores, generando así un derecho de las decisiones judiciales. En todos los sistemas jurídicos, también en el nuestro, hay alguna necesidad de determinar lo que en el derecho está relativamente indeterminado y en todos los sistemas parte de esa resolución debe ser llevada adelante por los tribunales, pues es a ellos a quienes les toca resolver ciertos asuntos en que esa indeterminación se hace patente. Al resolverlo, no necesariamente generan un precedente fuerte en el sentido del common law, pero sí determinan la forma en que se aplicará el derecho en ese caso, lo que típicamente modifica la forma de actuar de los vinculados por las reglas determinadas judicialmente, tanto en el caso concreto (pues están vinculados por la sentencia) como en otros similares que tengan posibilidades de ser llevados a ese tribunal o a otro que sea posible o probable que aplique un criterio similar (pues no desean exponerse a una sentencia desfavorable futura y, en el mejor de los casos, desean conformar su actuar con el derecho en la forma en que este es dicho por sus intérpretes más autorizados). Dado que el derecho no puede determinar todo para todos los $\operatorname{casos}^{31}$, existen aspectos que deben ser determinados en casos más concretos, sea por la administración o sea por el Poder Judicial.

31 La razón de esto es que el lenguaje en general, y el lenguaje jurídico en particular, tienen una "textura abierta". Así, H.L.A. Hart afirma: "Cualquiera sea el dispositivo, precedente o legislación, que se utilice para comunicar estándares de conducta, estos, independiente de lo bien que funcionen en el grueso de los casos ordinarios, en algún punto en que su aplicación esté en duda, se demostrarán indeterminado. Tendrán lo que se ha denominado una 'textura abierta”. En: Hart, H.L.A. (2012) The concept of law. $3^{\text {a }}$ edición. Oxford: Oxford University Press, 389 pp., p. 128. Antes habla de un "margen de vaguedad, o 'textura abierta” que tiene toda regla. Véase: Hart (2012) 123. Para un estudio completo sobre la vaguedad en el derecho, argumentando que la vaguedad es una característica que impregna todo el derecho, véase: EndicotT, Timothy (2001) Vagueness in law. Oxford: Oxford University Press, 226 pp. Más aún, la determinación completa de las normas no es siempre una deficiencia. Pese a las claras ventajas de regular importantes aspectos de la vida social por adelantado, y con importante precisión, también algún nivel de vaguedad de las normas jurídicas es deseable. Esto por las razones que aportaba el mismo Hart, a saber: nuestra relativa ignorancia de los hechos (no podemos predecir completamente toda situación, y por lo mismo no es razonable anticiparlas mediante normas jurídicas) y la relativa indeterminación de los objetivos (no podemos predecir todas las consideraciones relevantes que pueden estar juego en la aplicación de una norma) aconsejan que algo de la regulación de un asunto quede para ser determinado por quien debe aplicar dicha regulación en un caso concreto, con todos los antecedentes del caso a la vista. Véase: Hart (2012) 128-129. La vaguedad también puede ser una forma de evitar que una regulación se vuelva tan compleja y detallada que la determinación de la norma se vuelva contraproducente, en el sentido de que, en vez de hacer más claras las exigencias del derecho, las vuelva más confusas u oscuras. Véase: ENDicotT (2001) 190. 
Ahora bien, la determinación del derecho se realiza progresivamente por distintos órganos y de esta forma el derecho va ganando especificidad y mutando para adaptarse a nuevas circunstancias. El tribunal típicamente debe determinar cosas que aparecen vagas en las normas que aplica, o que simplemente no están resueltas por dichas normas, o que están determinadas pero por distintas categorías jurídicas las cuales pueden ser armonizadas de distintas maneras. La labor del tribunal es "decir el derecho", para lo cual aplica el derecho vigente, y aclara cuál es la forma en que este debe ser entendido, lo que implica resolver las fuentes de incerteza recién mencionadas: aclarar las prescripciones obscuras para el tipo de caso que se resuelve, resolver lo que no está resuelto, y determinar la armonización correcta de distintas categorías que pueden aplicar al caso. Todo esto debe ser fundamentado debidamente por el tribunal, aclarando las razones de su determinación. En realidad estas determinaciones del derecho que realiza el tribunal se cristalizan principalmente en la fundamentación de la sentencia: es ahí donde se formula la ratio, el principio con el que se resuelve el caso, el cual puede ser aplicado como tal en otros casos similares. Es ese principio el que puede implicar una determinación del derecho que va más allá de la situación concreta que está reglando el tribunal, y que por su generalidad se presta para otorgar una pauta de acción para situaciones similares.

En el caso ideal, el tribunal, al decidir un caso, deja al derecho mejor que como lo recibió, contribuyendo así a realizar el ideal del Estado de Derecho. Ese mejoramiento se produce en la medida que la sentencia: (a) sujetó a la autoridad efectivamente al derecho, lo que junto con dar vigor al derecho -validando así la expectativa de que este será cumplido- realiza en principio la primera idea constitutiva del Estado de Derecho; (b) permitió hacer más comprensibles las exigencias del derecho que vinculaba a la autoridad, lo que realiza la segunda idea del Estado de Derecho; al hacer esto último, (c) se vuelven más claros los límites de lo que está dentro y lo que está fuera del derecho y, de este modo, la primera idea de Estado de Derecho también queda realizada, no solo en principio, sino plenamente, pues siguen existiendo reglas capaces de guiar la conducta que efectivamente sujetan a la autoridad, ahora con mayor precisión y claridad que antes del fallo.

Pero también es posible que la intervención judicial sea disruptiva del ideal del Estado de Derecho y esto por medio de perjudicar directamente los valores vinculados a la segunda idea de Estado de Derecho. Al decir el derecho, un tribunal podría hacer más oscuras las exigencias del derecho, esto es, puede hacer más inciertos los límites que separan lo que es de lo que no es conforme a derecho. Son muchas las formas en que esto puede ocurrir. Por de pronto, al desarrollar el derecho, el tribunal 
puede incorporar elementos que incrementan la discrecionalidad propia o ajena, o que establecen exigencias inciertas. Al interpretar el derecho vigente los tribunales pueden dar versiones ambiguas o excesivamente vagas de las exigencias del derecho, o versiones excesivamente cambiantes a lo largo del tiempo. Asimismo, al armonizar distintas categorías, los tribunales pueden hacerlo de forma tal de subordinar la aplicación de las categorías precisas a las vagas, de forma tal de que la certeza dada por las primeras quede supeditada a la incerteza de las segundas. Así, los tribunales pueden arrojar dudas sobre toda la institucionalidad que establece exigencias determinadas al reprochar actos realizados conforme a la misma sin establecer criterios que permitan entender cuándo esa institucionalidad será corregida por tribunales y cuando no. Finalmente, las sentencias pueden ser escasamente fundadas en circunstancias en que los resultados de las mismas no son obvios y, por lo mismo, pueden aumentar la obscuridad acerca de las exigencias jurídicas que finalmente aplicarán a un caso.

No corresponde a este trabajo ofrecer un catálogo exhaustivo de las formas en que una sentencia puede perjudicar el Estado de Derecho. El punto es aclarar que existe una relación problemática entre la función de decir el derecho y el ideal de someter a las autoridades al imperio de un derecho que provea guía eficaz y cierta a las personas y a las autoridades. Es deseable que los tribunales, al decidir los casos de su competencia, realicen ellos mismos los criterios que permiten preservar al ordenamiento jurídico en buena forma, haciendo posible el que todos puedan guiar ordenadamente su conducta de acuerdo al derecho y así satisfacer mejor sus demandas. Eso impone exigencias al tribunal que van más allá de resolver correctamente. También exige resolver de forma tal de hacer más nítidas las exigencias del derecho en un área determinada y en ningún caso oscurecerlas.

\section{4) MEJORAR O EMPEORAR LA INSTITUCIONALIDAD COMO ESTÁNDAR DE LAS DECISIONES JUDICIALES}

Lo que hemos dicho implica un estándar para las decisiones de los tribunales de justicia. Cuando este estándar, descrito en el apartado anterior, es satisfecho por el fallo de un tribunal, entonces ese fallo habrá dejado la institucionalidad en mejor forma jurídica que como la recibió. Por el contrario, cuando el estándar no fue satisfecho, el tribunal habrá dejado una institucionalidad funcionando de forma más deficiente de como la recibió, esto es, más incierta, menos capaz de ordenar la acción humana y, por tanto, de limitar la discreción de la autoridad. Este estándar es, por supuesto, también un estándar para la evaluación de los fallos, 
en particular su impacto en el ordenamiento jurídico y en aquella parte de la actividad humana regida por las instituciones jurídicas implicadas en el pronunciamiento de cada sentencia. Como se nota, este no es un estándar sustancial: es indiferente respecto de si, por ejemplo, cierto derecho debe ser protegido con mayor o menor intensidad en ciertos casos, o si cierta regulación debe ser más o menos estricta, etc. Todas estas posiciones son compatibles en principio con el estándar propuesto. Asimismo, este criterio es indiferente respecto de si las normas deben ser modificadas en cierta área. Tanto permanencia como cambio son en principio compatibles con el estándar.

Lo que este criterio sí exige es una forma de realizar los cambios normativos, de adoptar ciertas mejoras en las instituciones o de implementar la interpretación correcta de una cierta institución. El punto no está en la substancia de lo que se decide, sino en la forma de la decisión judicial: una forma que haga prístinos los criterios que consideró el tribunal y las exigencias jurídicas que se entiende que rigen a los involucrados. El fallo debe dejar a los ciudadanos y a la autoridad con una institucionalidad igual o más determinada respecto de qué cosas son exigidas de ellos y de la autoridad, y en general en qué situación están desde la perspectiva del derecho. Para esto, el tribunal debe controlar la aplicación y la fidelidad al derecho vigente (y ser fiel él mismo al derecho), dar razones de sus decisiones suficientes para entender lo que las justifica e interpretar o, más generalmente, desarrollar el derecho cuando corresponda de forma tal de hacerlo más determinado.

Por cierto, este estándar se suma a otros para evaluar un fallo (su consistencia con el derecho en términos sustantivos, su justicia, su recta apreciación de los hechos, su adecuada deferencia o intervención, etc.) y obra en consonancia con ellos. No es el único estándar, ni el único asociado a la idea de Estado de Derecho. Por de pronto, hoy muchos entienden que la idea de Estado de Derecho ha cambiado desde un Estado de Derecho formal, con énfasis en la legalidad, a un Estado de Derecho sustantivo (o Estado Constitucional de Derecho), con énfasis en el respeto por principios y valores constitucionales sustantivos ${ }^{32}$. Aquí no discutimos esta preocupación por valores constitucionales ni su incorporación a la idea de Estado de Derecho. Tampoco discutimos si el nombre "Estado de Derecho" se aplica más propiamente a un tipo de exigencias, o a otras ${ }^{33}$.

32 Véase en este respecto, por ejemplo: Marshall, Pablo (2010) "El estado de derecho como principio y su consagración en la Constitución Política”. Revista de Derecho de la Universidad Católica del Norte, Año 17, $\mathrm{N}^{\circ}$ 2, pp. 185-204.

33 En relación a este punto, Raz críticamente afirma: "Debe insistirse en que el Estado de Derecho es solo una de las virtudes que un sistema jurídico puede poseer y por las cuales ha de ser juzgado. No debe ser confundido con la democracia, la justicia, la igualdad (ante la 
El punto es que aun cuando se entienda que la noción de Estado de Derecho ahora implica elementos que van más allá de los elementos formales, tradicionales del Estado de Derecho, el tipo de consideraciones explicadas en esta sección (las dos ideas constitutivas del Estado de Derecho asociadas con Dicey y Fuller) siguen siendo moral y políticamente relevantes, aun cuando a estas se agreguen otras cosas relevantes. Independiente de si, por ejemplo, se espera que el fallo de un tribunal refleje, además de la legalidad vigente, ciertos valores y principios constitucionales, sigue siendo cierto que dicho fallo tiene la capacidad de hacer al derecho más determinado, y por tanto más capaz de ordenar la acción de gobernados y autoridades así como de limitar la discreción de estas mismas, o de hacerlo menos determinado, y por tanto menos capaz de lograr esto mismo. Y sigue siendo deseable e importante que el juez haga lo primero y no frustre la capacidad del derecho de ordenar la conducta humana haciéndolo incierto, ni desmonte la capacidad del derecho de limitar la discrecionalidad de las autoridades, incluyendo la propia. Si además se espera que la comprensión y determinación de este derecho realizada por el juez responda a ciertos valores, esto es una exigencia adicional que no desmiente la anterior ${ }^{34}$.

ley o de otro tipo), derechos humanos de cualquier tipo, o respeto por la persona o por la dignidad del hombre". Véase: Raz (1979) 211.

En este sentido, Grégoire Webber ha entendido que las exigencias del Estado de Derecho operan al nivel de los medios (del derecho como medio para la realización de ciertos valores) mientras que otras exigencias (por ejemplo, respeto por los derechos) operan al nivel de los fines. Esto refuerza la idea de que si bien estas exigencias son asociadas todas a la idea de Estado de Derecho, son exigencias independientes. Véase Webber (2013) 399. Nuestro trabajo debe mucho a este iluminador ensayo de Webber. Con todo, se distingue en dos puntos. En primer lugar, la tesis de Webber es que la adjudicación de derechos humanos o fundamentales tiende a ser contraria a los desiderata de Fuller, particularmente mediante el uso del test de proporcionalidad. Nuestro análisis es más amplio, y pretende mostrar un modo general en que la intervención judicial puede afectar dichos desiderata, y en general hacer al derecho más indeterminado. Segundo, más allá de la indudable solidez del argumento de Webber, nosotros acá no argumentamos que la adjudicación de derechos fundamentales ni el uso del test de proporcionalidad implique o tienda a producir los defectos en términos de Estado de Derecho que han sido expuestos en esta sección. Sostenemos que también en la aplicación de derechos fundamentales relativamente abstractos es posible alcanzar el estándar que proponemos (Webber propone alternativas a la proporcionalidad en que esto es posible en la sección IV de su trabajo), sin hacer un juicio sobre si dicho estándar es incompatible con el uso de algún test para la adjudicación de derechos fundamentales tal como el test de proporcionalidad. Los autores han tratado la tensión entre proporcionalidad y la necesidad de sujetar la discrecionalidad judicial en: Urbina, Francisco Javier (2012) "A Critique of proportionality". American Journal of Jurisprudence, Vol. 57, pp. 49-80, pp. 66-80; y Urbina, Francisco Javier (2014) "Is it really that easy? A critique of proportionality and 'balancing as reasoning'". Canadian Journal of Law and Jurisprudence, Vol. 27, $\mathrm{N}^{\circ}$ 1, pp. 167-192, pp. 71-79. De la misma forma, los autores han tratado la tensión entre creación judicial de derechos "implícitos" y la necesidad de sujetar la discrecionalidad de ese proceso a las exigencias del Estado de Derecho tanto en el ámbito nacional como internacional en: 
También es, por cierto, posible que existan tensiones parciales entre la exigencia sustantiva de realizar ciertos valores o principios en la sentencia, y la exigencia formal (aunque moralmente relevante) de preservar la capacidad del derecho de ordenar la conducta humana. Parte del mérito de distinguir entre las distintas exigencias que hay en juego al hablar de Estado de Derecho es que permite ver que hay tensiones posibles entre las exigencias formales vistas en esta sección, y otras exigencias sustantivas. Agruparlas bajo un nombre no las hace armónicas automáticamente. La tensión más natural es aquella en que la aplicación de la institucionalidad vigente pareciera infringir lo que al juez le parece que es un valor constitucional sustantivo. Esta tensión es parcial porque en este caso la tensión radica solamente en que el juez debe optar entre aplicar la institucionalidad vigente, reforzando su vigencia, o aplicar el valor constitucional. Pero aun suponiendo que hiciera lo segundo, igual nada impide al juez el precisar exactamente en qué punto la institucionalidad compromete más allá de lo aceptable el valor constitucional, y qué partes de la institucionalidad están comprometidas y como, y qué partes no lo están.

En definitiva, nada impide al juez fundamentar debidamente su fallo y formular una ratio más o menos precisa que clarifique qué tipo de instituciones o acciones están siendo consideradas inconstitucionales y las consecuencias exactas que se deriva de ello. En este punto la labor de los tribunales de justicia ha sido en ocasiones problemática. Por ejemplo: tratándose de la resolución de numerosos recursos de protección, la práctica de algunos tribunales de no explicar en su fallo la forma exacta en que una medida viola un derecho constitucional específico, contentándose simplemente con constatar una ilegalidad y luego meramente enunciar una serie de derechos constitucionales (todos derechos altamente abstractos) que estarían siendo vulnerados, hace que el derecho aplicable sea menos nítido de lo que lo sería sin dichos fallos, y compromete innecesariamente los valores de Estado de Derecho mencionados. Más allá de cuál es el balance adecuado entre exigencias formales y sustanciales, cabe hacer notar que exactamente la misma protección a dichos derechos constitucionales, aún interpretados de la forma más expansiva, sería consistente con el otorgar a los fallos un fundamento adecuado en todos los puntos

Candia, Gonzalo (2014) "Analizando la tesis de los derechos implícitos: comentario a la sentencia del Tribunal Constitucional recaída sobre el requerimiento de inaplicabilidad rol No. 2.408-2013". Revista de Derecho de la Universidad Católica del Norte, Vol. 21, N 1, pp. 497-521, pp. 512-514 y Candia, Gonzalo (2015) "Derechos implícitos y Corte Interamericana de Derechos Humanos: una reflexión a la luz de la noción de Estado de Derecho". Revista Chilena de Derecho, Vol. 42, N³, pp. 873-902, respectivamente. 
necesarios para fundar un recurso de protección, y con precisar cuál es el derecho directamente involucrado. Estas deficiencias en la forma en que los tribunales pueden controlar el apego a derecho de otras autoridades merecen mayor atención, pues es un vicio que se observa en algunos fallos de nuestros tribunales. Proveer una ilustración de como estas deficiencias pueden manifestarse en nuestro derecho es el objeto de la siguiente sección.

\section{5) Ilustración: CASO EL MORRO II}

En el presente artículo, se ha buscado: (a) explicar como algunas exigencias específicas asociadas al Estado de Derecho impactan el proceso adjudicatorio, definiendo estándares específicos para las decisiones judiciales, y (b) demostrar como las decisiones judiciales pueden poner en tensión ambas exigencias del Estado de Derecho, aun cuando aparezca que en principio lo promueven por sujetar a la administración a una norma jurídica. Desde esa perspectiva, es posible afirmar que el Estado de Derecho no solo dirige sus exigencias al legislador, sino también al juez. Ello en la medida que los requerimientos del Estado de Derecho tienen efectos sistémicos dentro del derecho y, por tanto, "se aplican a todo lo largo y a todo lo ancho de todas las operaciones básicas de un sistema jurídico" 35 , lo que incluye, naturalmente, los procesos de adjudicación.

Sin embargo, tal como se analizó en la primera parte de este trabajo, existe la posibilidad de que los adjudicadores, por medio de sentencias que no se ajusten a los criterios descritos, generen tensiones internas dentro de las exigencias propias del Estado de Derecho. Ello se produce cuando los tribunales, por medio de sus fallos, disminuyen la capacidad de la institucionalidad de guiar la acción humana y de limitar la discreción de la autoridad.

A continuación, se ilustrarán estas tensiones por medio del análisis de una sentencia dictada por la Corte Suprema en el contexto de la resolución de un recurso de protección recaído sobre el caso Comunidad Agricola Los Huascoaltinos con Comisión de Evaluación Ambiental de III Región de Atacama. El fallo en cuestión permitirá mostrar la tensión entre Estado de Derecho y control jurisdiccional de la autoridad descrita en la primera

35 Summers, Robert (2002) "Los principios del estado de derecho" (Pablo Larrañaga, trad.). En Carbonell, Miguel; Orozco, Wistano; y Vásquez, Rodolfo (directores): Estado de Derecho. Concepto, fundamentos y democratización en América Latina. Ciudad de México: Siglo XXI Editores, 384 pp., pp. 37-60 y p. 44. 
parte de este trabajo. Dicha tensión se produce, básicamente, debido al uso por parte de la Corte Suprema de estándares cuya indeterminación normativa crea espacios de incerteza y vaguedad en la aplicación de una institucionalidad más precisa y, por tanto, determinada. La indeterminación generada por el fallo es de una magnitud suficiente como para restar guía jurídica necesaria a quienes están sujetos por la institucionalidad comprometida para ordenar su actividad conforme a ella -restando así eficacia a la misma institucionalidad, incluidas las exigencias contenidas en los mismos fallos comentados-, y haciendo más incierto los límites que limitan la discrecionalidad de las autoridades involucradas -jueces incluidos-. Así, el caso presenta un buen ejemplo de la situación que hemos explicado en las secciones precedentes. Este fallo nos parece especialmente apto para ilustrar las deficiencias que hemos mencionado en este artículo pues presenta un contraste entre una institucionalidad altamente determinada (la institucionalidad ambiental) y por tanto apta para otorgar bastante guía jurídica a los sujetos por ella y a quienes la aplican, y por otro ciertos derechos fundamentales abstractos que son utilizados para evaluar los resultados de la primera. En este caso, el contraste es especialmente agudo y por tanto ejemplifica de manera más clara como la revisión judicial puede ampliar la vaguedad e indeterminación del derecho.

De acuerdo al artículo 20 de la Constitución, el recurso de protección es un instrumento de naturaleza cautelar, que busca restablecer "el imperio del derecho y asegurar la debida protección al afectado" en aquellos casos en que existiere una "privación, perturbación o amenaza en el ejercicio legítimo" de ciertos derechos y garantías constitucionales. El estándar judicial aplicable para la resolución del caso es el contenido en el artículo 20 inciso segundo, en la medida que los recurrentes alegaron la infracción del artículo 19 No 8 de la Constitución, esto es, el derecho a vivir en un medio ambiente libre de contaminación.

Un grupo de comunidades indígenas diaguitas hicieron uso de esta acción constitucional para solicitar, primero a la Corte de Apelaciones de Copiapó y después a la Corte Suprema, que invalidara la resolución exenta $N^{\circ} 232$ de calificación ambiental (en adelante, RCA) dictada por la Comisión Ambiental de la Región de Atacama con fecha 22 de octubre de 2013. Dicha resolución había aprobado la realización del proyecto minero El Morro, proyecto llevado adelante por la empresa Goldcorp.

Este recurso es parte de una tortuosa historia, la cual comienza el año 2012, cuando la propia Corte Suprema invalidó la resolución exenta N 49 dictada por la Comisión de Evaluación de la Región de Atacama, 
la cual había calificado favorablemente la realización del proyecto ${ }^{36}$. En dicha oportunidad la razón invocada por la Corte para paralizar la continuidad del proyecto El Morro fue que no se practicó la consulta exigida por el Convenio $\mathrm{N}^{\circ} 169$ de la OIT respecto de las comunidades indígenas que podían verse afectadas por el proyecto. A partir de la referida decisión, tanto los órganos competentes de la Administración como la empresa procedieron a efectuar esa consulta, tras la cual la Comisión Ambiental de la región procedió a autorizar el desarrollo de la iniciativa.

Fue esa resolución, que por segunda vez autorizaba a la empresa a proceder a realizar el proyecto Los Morros, la que fue objeto del recurso de protección en cuestión. El argumento invocado por la recurrente fue similar al del caso anterior: básicamente, (a) que la consulta practicada a la comunidad huascoandina no cumplía las condiciones impuestas tanto por el Convenio 169 como por la sentencia anteriormente pronunciada por la Corte Suprema y (b) que ciertas asociaciones, comunidades indígenas diaguitas y personas afectadas no habían sido incluidas en el proceso de consulta indígena practicado ${ }^{37}$.

El recurso de protección deducido ante la Corte de Apelaciones de Copiapó fue rechazado unánimemente a través de una sentencia dictada el 28 de abril de $2014^{38}$. Dicha sentencia fue apelada ante la Corte Suprema que, finalmente, acogió el recurso.

Los recurrentes arguyeron que la resolución exenta $N^{\circ} 232$ infringía una serie de garantías constitucionales, tales como aquellas comprendidas en el artículo $19 \mathrm{~N}^{\circ} 2,8,21$ y 24 de la Constitución, sin especificar mayormente cuáles serían las razones de dicha afectación ${ }^{39}$.

En este caso, como se ha dicho, el afectado principal con la interposición del recurso había sido impedido anteriormente de proceder a la ejecución del proyecto, pese a la autorización de todos los órganos adscritos al Servicio de Evaluación Ambiental. La razón de ello era bien precisa:

36 Corte Suprema. 27 de abril de 2012. Rol No 2211-2012. "Comunidad Agrícola Los Huasco Altinos con Comisión de Evaluación Ambiental de III Región de Atacama”. Disponible en: http://decs.pjud.cl/Documentos/Fallos/2.211-2012.pdf [fecha de visita 2 de febrero de 2016].

37 Corte Suprema. 7 de octubre de 2014. Rol No 11.299-2014. "Comunidades Indígenas Diaguitas Yastai de Juntas Valeriano y otros con Comisión de Evaluación Ambiental de III Región de Atacama”. Disponible en: http://decs.pjud.cl/Documentos/Jurisprudencia/Fallos\%20Tercera\%20Sala\%20Nueva\%20institucionalidad\%20ambiental.pdf [fecha de visita 7 de septiembre de 2015], pp. 2-3.

38 Corte de Apelaciones de Copiapó. 28 de abril de 2014. Rol No 436-2013. Disponible en: http://cortes-apelacion.vlex.cl/vid/comunidad-indigena-diaguita-yastai-571488430 [fecha de visita 7 de septiembre de 2015].

39 CS. Rol N¹1.299-2014, pp. 10-11. 
el incumplimiento de la consulta indígena. La misma se había efectuado, pese a que la comunidad huascoandina no quiso continuar con el proceso. A pesar de ello, la CONADI, la Corporación Nacional Indígena, había aprobado los términos en los cuales la consulta fue efectuada, razón por la cual la Comisión Ambiental de Atacama había dictado la RCA aprobando el inicio del proyecto.

En su fallo, la Corte Suprema primero define el contorno de su propia competencia. En efecto, la Corte señala que, en principio, corresponde a los tribunales medioambientales, en cuanto órganos especializados, pronunciarse en torno a materias de fondo como la solicitud de invalidación de una RCA en conformidad a lo dispuesto en los artículos 29 y 30 bis de la Ley $\mathrm{N}^{\circ} 19.300^{40}$. Luego, la Corte establece una excepción: podrá recurrirse a los tribunales ordinarios (Cortes de Apelaciones y Corte Suprema) únicamente cuando "las ilegalidades denunciadas [por la afectada] requier[an] de una cautela urgente e inmediata [respecto de] las garantías constitucionales que [se denuncian] como infringidas" ${ }^{1}$.

La Corte, pues, invocó un estándar de juicio que permite determinar cuándo los casos vinculados con la invalidación de una RCA deben ser excepcionalmente conocidos por tribunales ordinarios a través de un recurso de protección. Ello ocurre cuando de los hechos del caso se puede entender que existe la necesidad de brindar una cautela urgente $e$ inmediata de garantías constitucionales a los potenciales afectados por la implementación de la RCA. La Corte genera un estándar más o menos determinado para resolver el punto, lo que va, precisamente, en la línea de las exigencias del Estado de Derecho. Sin embargo, la Corte Suprema, tras describir el estándar, no analiza si de los hechos de la causa sujeta a su conocimiento aparece la necesidad urgente e inmediata de otorgar protección a garantías constitucionales específicas. Por el contrario; la Corte, tras enunciar el criterio, entra derechamente en el análisis del fondo del caso $^{42}$. Al no expresar por qué en este caso existe tal necesidad, la Corte hace incierto su propio criterio, al arrojar dudas sobre como este aplica - ¿por qué aplica a este caso, en que no es evidente que haya tal necesidad urgente e inmediata?-, o si aplicó al caso otro criterio no explicitado por la Corte.

En su análisis de fondo, la Corte Suprema se limitó a indicar que la CONADI no había justificado suficientemente las razones de por qué la consulta realizada a la comunidad huascoandina era apropiada y de por

\footnotetext{
$40 \quad$ CS. Rol No 11.299-2014, p. 5.

$41 \quad$ CS. Rol N ${ }^{\circ} 11.299-2014$, p. 7.

42 CS. Rol N ${ }^{\circ} 11.299-2014$, p. 7.
} 
qué la misma no había incorporado a otras asociaciones. Esta falta de motivación constituía una ilegalidad, dadas las exigencias de fundamentación de los actos administrativos que afecten derechos, así como de las resoluciones, establecidas respectivamente en los artículos 11 inciso segundo y 41 inciso cuarto de la ley 19.880 (Ley de Bases de los Procedimientos Administrativos), así como la exigencia de "pronunciamientos ambientales fundados" de los artículos $9^{\circ}$ y $9^{\circ}$ bis de la ley 19.300 (Ley sobre Bases Generales del Medio Ambiente). Esta falta, sostiene la Corte, "deviene en que la opinión favorable otorgada al proyecto adolezca de un vicio que la transforma en ilegal y arbitraria" ${ }^{43}$. Asimismo, el acto terminal recurrido en autos, la RCA, adolecería de los mismos vicios de ilegalidad y arbitrariedad $^{44}$.

Pese a emitir ese argumento, la Corte no refirió con precisión como esa supuesta falta de motivación impactaba el ejercicio de los derechos fundamentales argüidos por la parte recurrente. Recuérdese que el recurso de protección exige que se establezca no solo que el acto u omisión recurrido sea ilegal o arbitrario, sino también que dicha arbitrariedad o ilegalidad afecte alguno de los derechos mencionados en el artículo 20 de la Constitución, implicando una privación, perturbación, o amenaza de los mismos. El recurso de protección no protege contra toda ilegalidad o arbitrariedad (de ser así abarcaría el ámbito de prácticamente toda acción procesal), sino solo de aquellas que implican una afectación de un derecho en los términos del artículo 20 mencionado. La Corte simplemente en el final del fallo se limita a señalar que la RCA en cuestión "deviene en ilegal y arbitraria por carecer del sustento que la ley le impone" 45 , lo que vulneraría el artículo $19 \mathrm{~N}^{\circ} 2$ de la Constitución por cuanto quebraría la relación de igualdad entre aquellas comunidades respecto de las cuales se pronunció expresamente la CONADI negando la realización de la consulta ${ }^{46}$.

Pero este argumento dista de ser concluyente, toda vez que no aborda precisamente el punto crucial a la hora de establecer si una diferenciación es arbitraria o no, y por tanto proscrita por el artículo $19 \mathrm{~N}^{\circ} 2$ de la Constitución: esto es, si la agrupación realizada por la autoridad -es decir, la diferenciación en diversas categorías y la incorporación de diver-

43 CS. Rol N 11.299-2014, p. 25. Con todo, en sentido estricto la Corte solo ha establecido la ilegalidad del acto. Dado que el recurso de protección exige establecer o la ilegalidad, o la arbitrariedad (o ambas), no hay consecuencias prácticas de establecer solo uno de estos vicios en vez de ambos.

$44 \quad$ CS. Rol No $11.299-2014$, p. 25

45 CS. Rol No $11.299-2014$, p. 25.

46 CS. Rol No 11.299-2014, pp. 25-26. 
sas entidades a las distintas categorías- es razonable o no. Como esto es independiente de la fundamentación de la medida (puede ser razonable pero no fundamentada, o fundamentada pero no razonable), el solo establecer que la medida no fue fundada no establece nada, aun respecto de si la medida realiza una agrupación no razonable, y por tanto discrimina arbitrariamente en los términos proscritos por el artículo $19 \mathrm{~N}^{\circ} 2$ de la Constitución. El fallo correspondiente al caso Los Morros II se limita a indicar que ha existido una infracción del artículo $19 \mathrm{~N}^{\circ} 2$ de la Constitución estableciendo un cartabón de comparación extrañísimo en relación al juicio de igualdad aplicado: aquellas comunidades indígenas (¡todas?) respecto de las cuales la CONADI ha negado fundadamente la realización de la consulta al tenor de lo dispuesto en el Convenio $\mathrm{N}^{\circ} 169$. Asimismo, la Corte no efectúa ningún juicio respecto del resto de los derechos constitucionales cuya infracción alega la recurrente.

Dado que no hay un razonamiento desarrollado sobre el particular, la forma como el tribunal llega a la conclusión en el asunto principal cuya decisión se reclama, es opaca. Lo mismo ocurre, como se vio, respecto del asunto adjetivo sobre qué tribunal corresponde que vea este tipo de asuntos: si el tribunal ambiental mediante las acciones pertinentes, o las Cortes mediante el recurso de protección (un punto no menor, dado que involucra el derecho fundamental al juez natural). Al no estar especificado como el tribunal llega a las conclusiones mencionadas en esos puntos importantes, no queda claro entonces bajo qué condiciones, por ejemplo, corresponde ejercer la acción de protección y bajo qué otras condiciones las acciones pertinentes en el Tribunal Ambiental. Ni queda claro bajo qué condiciones el derecho del artículo $19 \mathrm{~N}^{\circ} 2$ de la Constitución queda comprometido, aparte de las condiciones específicas de este caso. No aparece un principio, ratio, que tanto autoridades como particulares puedan seguir para tener claridad respecto de como deben ordenar su acción conforme a derecho.

Tan significativo para la promoción del Estado de Derecho es lo que dice como lo que no dice el juez. En efecto, los silencios del adjudicador pueden generar espacios de indeterminación en la institucionalidad. Los Morros II es uno de esos casos. La institucionalidad ambiental es, entre otras cosas, una forma altamente determinada de especificar el alcance de derechos fundamentales que pueden estar implicados en la realización de un proyecto que tenga un impacto ambiental significativo - particularmente, pero no solamente, el derecho a vivir en un medio ambiente libre de contaminación-, así como otros valores relevantes. El sentido de tener una institucionalidad que sea así de determinada, en vez de dejar que los 
operadores jurídicos actúen basados simplemente en valores constitucionales indeterminados, es en gran medida el mencionado en la sección precedente: favorecer la eficacia del derecho y su capacidad de ordenar la conducta de forma consistente con él mediante pautas de acción relativamente precisas, claras, prospectivas, coherentes, etc., es decir, pautas de acción que pueden ser adoptadas como guía de la conducta humana; y limitar, por lo mismo, la discreción de la autoridad, al vincularla a un derecho determinado cuyo contenido no está (o no completamente) a su vez sujeto a la determinación que haga la misma autoridad en el caso concreto, sino que vincula tanto a la autoridad como al gobernado. Pero ambas exigencias quedan comprometidas cuando la determinación que se ha hecho del derecho, por ejemplo, mediante una institucionalidad ambiental, queda totalmente supeditada a la aplicación de categorías jurídicas vagas y abstractas, aplicadas con escasa justificación y desarrollo por los tribunales.

En esto radica el problema del fallo, como ilustración del problema más general descrito en la sección 2 de este trabajo. Aun cuando la solución sea la correcta en términos de justicia sustantiva, o incluso de legalidad sustantiva, el fallo supedita la aplicación de una institucionalidad determinada a la aplicación vaga e impresionista de categorías jurídicas abstractas, tales como los derechos fundamentales. Al hacer esto, hace a la institucionalidad más indeterminada, toda vez que incluso la aplicación de sus partes determinadas está supeditadas a la aplicación de pautas altamente indeterminadas. Esta indeterminación, como hemos explicado, es problemática. Por lo tanto, el fallo deja a la institucionalidad, por decirlo de algún modo, peor que como la encontró. De haber ofrecido el tribunal una ratio precisa para la resolución de los asuntos sustantivo y adjetivo mencionados, el problema que mostramos no se habría presentado, o se habría presentado de forma mucho más tenue. Como es posible apreciar, el problema no es de fondo, sino de forma.

\section{ConClusión}

En el presente trabajo hemos intentado explicar como el control jurisdiccional de los actos de las autoridades puede ser disruptivo para el mismo derecho cuyo cumplimiento se busca controlar, y de esta forma para el ideal del Estado de Derecho. En efecto, explicamos dos ideas específicas asociadas al Estado de Derecho, las cuales establecen exigencias precisas y específicas. Estas exigencias pueden encontrarse en tensión, de 
tal forma que la realización de una perjudica el cumplimiento de la otra -lo que a su vez puede perjudicar indirectamente el cumplimiento futuro de la primera-. Esto puede ocurrir en la revisión judicial de los actos de una autoridad. Así, por ejemplo, ocurre cuando la actividad de los tribunales en cuestión hace más indeterminado el derecho cuya vigencia controla. Al hacerlo, el juez deja a ese derecho "en peor forma", esto es, menos capaz de orientar la acción humana por cuanto sus prescripciones se hacen menos claras. Esto trae como consecuencia una menor capacidad del derecho para limitar la discreción de la autoridad.

En efecto, al ser el derecho menos determinado, los límites que este fija a la autoridad se desdibujan -incluyendo los límites que el derecho establece a la acción de los mismos tribunales-. Ambos efectos indeseables de la indeterminación que la sentencia introduce en el derecho (el hacer menos determinado el derecho, y el desdibujar por tanto los límites que este impone a la discreción de las autoridades) son, como se dijo, de gran relevancia política y moral. Por de pronto, resienten el tipo de forma de relación que el ideal del Estado de Derecho busca generar entre autoridades y gobernados: una relación que no es de total sumisión de los segundos a la voluntad irrestricta de los primeros, sino de una coordinación de ambos para el bien común en base a normas comunes. Asimismo, al ser una parte de la institucionalidad menos determinada, el derecho se hace menos efectivo, pues es menos capaz de ordenar la conducta humana de forma consistente con los fines de dicha institucionalidad. Un tercer problema es que la falta de certeza respecto de como será tratada una acción por los órganos que aplican el derecho, afecta la posibilidad de las personas de predecir los efectos de sus acciones y realizar proyectos de vida más complejos que exigen planificación. Y, por último, se perjudica la necesaria coordinación social realizada por el derecho, la cual es posible precisamente gracias a la capacidad del derecho de determinar cursos de acción para toda la sociedad o partes de ella. Es, por tanto, deseable que los tribunales asuman como un estándar de su actuación al decidir las causas bajo su competencia el no hacer más indeterminado el derecho que aplican y, en lo posible, hacerlo más determinado cuando ello fuese necesario. Cuando no se cumple este estándar, existe un defecto en la institucionalidad, el cual tiene las consecuencias y relevancia que hemos mostrado. Este defecto se presenta en algunos casos resueltos por nuestros tribunales, y este artículo ilustra como dicho efecto se manifiesta en una sentencia de la Corte Suprema chilena en materia medioambiental.

Como es posible apreciar, este artículo analiza un defecto de forma. Nuestra crítica no apunta al fondo de lo resuelto por tribunales: no 
discute si la decisión de algún tribunal es justa en cuanto a la adjudicación misma, ni si reposa en la mejor interpretación de nuestro derecho. Nuestro argumento tampoco es ni implica per se una crítica a la creación judicial de pautas generales de derecho (y no solo para el caso concreto), ni a la aplicación de derechos abstractos por parte de los tribunales, y ni siquiera al llamado "activismo judicial”. El estándar que se propone puede ser en principio cumplido por una sentencia que caiga en todas esas categorías. Solo exige que los tribunales al hacerlo no hagan la institucionalidad jurídica que aplican al caso concreto más indeterminada de lo que es $y$, de aplicar categorías indeterminadas, que la sentencia las determine en la medida de lo posible, mostrando exactamente qué es lo que dichas categorías exigen para el tipo de casos como el de la especie. Esto exige, entre otras cosas, muy centralmente que el fallo contenga un razonamiento desarrollado que articule con precisión una ratio que permita comprender la exigencia que el tribunal entiende que se presenta en casos de este tipo, como se incumple dicha exigencia, y cuáles son las consecuencias del incumplimiento.

Al cumplir este estándar en una sentencia, decimos que el tribunal deja el derecho mejor que como lo recibió. Cuando esto ocurre, los tribunales no solo hacen justicia en el caso concreto, sino que colaboran con el ideal (exigido por la justicia) de una sociedad regida no por la voluntad irrestricta de uno o más hombres sobre el resto sino, en la mayor medida posible y en último término, por el derecho.

\section{Bibliografía Citada}

Bingham, Tom (2011) The Rule of Law. Londres: Penguin Books, 230 pp. Bordalí, Andrés (2006) "La administración pública ante los tribunales de justicia chilenos". Revista Chilena de Derecho, Vol. 33, N 1, pp. 17-36.

Candia, Gonzalo (2014) "Analizando la tesis de los derechos implícitos: comentario a la sentencia del Tribunal Constitucional recaída sobre el requerimiento de inaplicabilidad rol N $\mathrm{N}^{\circ}$ 2.408-2013". Revista de Derecho de la Universidad Católica del Norte, Vol. 21, N 1, pp. 497-521.

Candia, Gonzalo (2015) "Derechos implícitos y Corte Interamericana de Derechos Humanos: una reflexión a la luz de la noción de estado de derecho". Revista Chilena de Derecho, Vol. 42, N 3, pp. 873-902.

CEA, José Luis (2005) "Estado constitucional de derecho: nuevo paradigma jurídico". Anuario Latinoamericano de Derecho Constitucional, Año 11, pp. 43-56. 
Craig, Paul (1997) "Formal and substantive conceptions of the rule of law: an analytical framework". Public Law, N467, pp. 467-487.

Dicey, A.V. (2013a) The law of the constitution. Oxford: Oxford University Press, 572 pp.

Dicey, A.V. (2013b) Comparative constitutionalism. Oxford: Oxford University Press, 402 pp.

EndicotT, Timothy (2001) Vagueness in law. Oxford: Oxford University Press, 226 pp.

EndicotT, Timothy (2012) “Legal interpretation”. En Marmor, Andrei (director): The Routledge companion to philosophy of law. New York: Routledge University Press, 656 pp., pp. 109-122.

FINNIS, John (2011a) Natural law and natural rights. 2a edición. Oxford: Oxford University Press, $512 \mathrm{pp}$.

Finnis, John (2011b) "Law as coordination". En Finnis, John (autor): Collected essays of John Finnis. Volume IV. Oxford: Oxford University Press, 509 pp., pp. 66-73.

Fuller, Lon (1969) The morality of law. 2a edición. New Haven: Yale University Press, 262 pp.

Hart, H.L.A. (2012) The concept of law. 3a edición. Oxford: Oxford University Press, 389 pp.

Marshall, Pablo (2010) "El estado de derecho como principio y su consagración en la Constitución Política”. Revista de Derecho de la Universidad Católica del Norte, Año 17, N², pp. 185-204.

Nogueira, Humberto (2009) "Los derechos económicos, sociales y culturales como derechos fundamentales efectivos en el constitucionalismo democrático latinoamericano”. Estudios Constitucionales, Año 2, No 7, pp. 143-205.

NúNEEZ, Juan Ignacio (2011) "Vigencia del dogma de la irresponsabilidad del estado legislador en el estado constitucional de derecho. Ensayando bases para una teoría sobre la responsabilidad patrimonial del estado legislador". Estudios Constitucionales, Año 9, $\mathrm{N}^{\circ} 1$, pp. 217-230.

Raz, Joseph (1979) "The rule of law and its virtue". En Raz, Joseph (autor): The authority of law: essays on law and morality. Oxford: Oxford University Press, 292 pp., pp. 210-229.

Rodríguez, Pablo (1990) Teoría de la interpretación jurídica. Santiago de Chile: Editorial Jurídica de Chile, 182 pp.

Silkenat, James R.; Hickey, James E.; y Barenboim, Peter D. (editores) (2014) The legal doctrines of the rule of law and the legal state (Rechtsstaat). Heidelberg: Springer, 367 pp.

Silva Bascuñán, Alejando (1997) Tratado de Derecho Constitucional. Principios, Estado y Gobierno. Tomo I. Santiago de Chile: Editorial Jurídica de Chile, 588 pp.

Soto-Kloss, Eduardo (1996) Derecho Administrativo - Bases Fundamentales. Santiago de Chile: Editorial Jurídica de Chile, Tomo II, 386 pp. 
Summers, Robert (2002) "Los principios del estado de derecho" (Pablo Larrañaga, trad.). En Carbonell, Miguel; Orozco, Wistano; y Vásquez, Rodolfo (directores): Estado de Derecho. Concepto, fundamentos y democratización en América Latina. Ciudad de México: Siglo XXI Editores, 384 pp., pp. 37-60.

Tamanaha, Brian (2004) On the rule of law. History, politics, theory. Cambridge: Cambridge University Press, 180 pp.

Urbina, Francisco Javier (2012) "A critique of proportionality". American Journal of Jurisprudence, Vol. 57, pp. 49-80.

UrbinA, Francisco Javier (2014) "Is it really that easy? A critique of proportionality and 'balancing as reasoning'". Canadian Journal of Law and Jurisprudence, Vol. 27, $\mathrm{N}^{\circ}$ 1, pp. 167-192.

Urbina, Francisco Javier y Urbina, Ignacio (2015) "Revisión judicial de la sanción ambiental (A propósito de Pascua Lama)". En Fermandois, Arturo y García, José Francisco (editores): Sentencias Destacadas 2014. Santiago de Chile: Libertad y Desarrollo, 377 pp., pp. 61-89.

WaLDRON, Jeremy (2011) "The rule of law and the importance of procedure". En Fleming, James E. (editor): Getting to the Rule of Law. Nueva York: New York University Press, 298 pp.

Webber, Gregoire (2013) "Rights and the rule of law in the balance". Law Quarterly Review, Vol. 129, pp. 399-419.

\section{JURISPRUDENCIA CITADA}

Corte Suprema. 27 de abril de 2012. Rol No 2211-2012. "Comunidad Agrícola Los Huasco Altinos con Comisión de Evaluación Ambiental de III Región de Atacama”. Disponible en: http://decs.pjud.cl/ Documentos/Fallos/2.211-2012.pdf [fecha de visita: 2 de febrero de 2016].

Corte Suprema. 7 de octubre de 2014. Rol No 11.299-2014. "Comunidades Indígenas Diaguitas Yastai de Juntas Valeriano y otros con Comisión de Evaluación Ambiental de III Región de Atacama". Disponible en: http://decs.pjud.cl/Documentos/ Jurisprudencia/Fallos\%20 Tercera \% 20Sala \% 20 Nueva $\% 20$ institucionalidad\%20ambiental.pdf [fecha de visita: 7 de septiembre de 2015]. 